\title{
La chasse au gène de la polykystose rénale autosomique dominante
}

Les 7 et 8 juin 1990 s'est tenue à Paris, au château de Longchamp, une réunion de 80 cliniciens, généticiens et biologistes moléculaires, consacrée aux plus récents progrès des travaux sur les polykystoses rénales héréditaires dominantes et récessives. Cette réunion était organisée par Micheline Lévy dans le cadre d'une action concertée européenne : Towards prevention of renal failure caused by inherited polycystic kidney disease, regroupant plus de 30 laboratoires de la CEE. Des groupes américains, finnois et israéliens ont également participé à la réunion. Les aspects cliniques et épidémiologiques aussi bien que la génétique moléculaire de cette affection ont été abordés. Avant d'exposer les résultats les plus récents, il nous semble utile de rappeler quelques données sur ces maladies.

La polykystose rénale autosomique dominante (ADPKD, autosomal dominant polycystic kidney disease) est une des plus fréquentes génopathies humaines, touchant environ une personne sur 700 à 1000 , tout au moins dans les pays occidentaux où des données épidémiologiques sont disponibles. Cette affection se manifeste par le développement de multiples kystes rénaux bilatéraux. Environ $50 \%$ des malades évoluent vers l'insuffisance rénale terminale, nécessitant dialyse et/ou transplantation, en moyenne autour de 50 ans, rarement avant 40 ans, parfois après 60 ou 70 ans. Il

Zerres (Bonn, RFA) et M.-F. Gagnadoux (Paris), des formes graves exceptionnelles, touchant le nourrisson et évoluant rapidement vers l'insuffisance rénale. Ces formes doivent être bien distinguées de la polykystose rénale autosomique récessive (ARPKD, autosomal recessive polycystic kidney disease) observée chez l'enfant. La diversité dans l'évolution de l'ADPKD est bien démontrée par l'étude épidémiologique faite récemment en Bretagne et les Pays-deLoire et organisée par P. Simon (Saint-Brieuc) H.F. Thébaud (Vannes) et al. D'autres études coopératives européennes sont en cours, portant notamment sur les facteurs déterminant la progression (M. Zeier, Heidelberg, RFA), l'atteinte chez les jumeaux (M. Lévy, Paris) et l'association à des anévrismes des artères cérébrales (Y. Pirson, Bruxelles, Belgique et D. Chauveau, Paris), complication rare et redoutable sur laquelle on dispose de peu de données épidémiologiques.

Le mécanisme de formation des kystes rénaux est jusqu'à présent inconnu. P.D. Wilson (Piscataway, NJ, États-Unis) a présenté des résultats fascinants sur un mécanisme potentiel : dans les cellules épithéliales des kystes, la $\mathrm{Na}^{+}, \mathrm{K}^{+}$-ATPase est mal localisée, située à la face apicale, alors que normalement elle n'est présente qu'à la face baso-latérale des cellules. Cela pourrait contribuer à l'accumulation de liquide dans les kystes. L'anomalie pourrait porter sur la sous-unité $\beta$, non catalytique, de la $\mathrm{Na}^{+}, \mathrm{K}^{+}$-ATPase, responsable de son ancrage à la membrane cellulaire. D'autres anomalies cellulaires, concernant notamment le cytosquelette, accompagnent la localisation anormale de la $\mathrm{Na}^{+}, \mathrm{K}^{+}$-ATPase.

Jusqu'en 1985 les connaissances fondamentales sur l'ADPKD et l'ARPKD n'ont que très peu progressé, en dépit d'énormes progrès dans le traitement des malades. C'est à cette date que $\mathrm{S}$. Reeders, $\mathrm{M}$. Breuning et al. [1] ont démontré une liaison génétique entre l'ADPKD et le locus $\alpha$-globine à l'extrémité du bras court du chromosome 16, grâce à l'utilisation d'un polymorphisme de restriction (RFLP, restriction fragment length polymorphism) de type VNTR (variable number of tandem repeats) (locus D 16 S85, sonde 3'HVR). Ce résultat a été très largement confirmé par la suite dans différents laboratoires du monde entier. Depuis cette première percée fondamentale, la recherche du gène se poursuit en appliquant toutes les stratégies de la génétique inverse qui ont déjà fait leurs preuves pour isoler les gènes responsables de la myopathie de Duchenne (DMD) (m/s, n० 3, vol. 4, p. 141), de la mucoviscidose (CF) $\left(\mathrm{m} / \mathrm{s}, n^{\circ} 8\right.$, vol. 5, p. 589) ou de la neurofibromatose de type 1 (NF1) (m/s, $n^{\circ} 8$, vol. $6, p .815)$ pour ne citer que les plus connus. Ces travaux ont amené à caractériser de nombreuses autres sondes génétiques de plus en plus proches encadrant le locus PKD1. 
L'étude de nombreuses familles affectées par l'ADPKD, nécessaire pour positionner ces sondes les unes par rapport aux autres et par rapport au locus de la maladie, a permis de découvrir qu'il existe au moins deux gènes, non liés génétiquement, responsables d'une maladie cliniquement identique $[2,3]$, la seule différence étant peut-être l'âge de l'insuffisance rénale terminale plus tardif pour la forme PKD2 [4] ; celle-ci représentent $5 \%$ à $10 \%$ du total des familles atteintes.

Parmi toutes les communications présentées (plus de 35), quatre nous apparaissent comme très importantes. Les travaux récents du groupe de $\mathrm{S}$. Reeders à Yale, États-Unis, ont permis d'établir la cartographie physique d'une région de 600 à 800 kilopaires de bases (kpb) contenant le locus PKD1 [5]. Le résultat le plus marquant de cette étude est que cette région du génome contient probablement au moins 25 gènes exprimés, comme l'indique la haute densité de dinucléotides $\mathrm{CpG}$ non méthylés (îlots HTF, Hpa2 tiny fragments) trouvée. Cela laisse supposer que le gène PKD1 est de petite taille, ce qui rend plus difficile la compréhension de la fréquence élevée de néo-mutation (de 6,5 à $10.10^{-5}$ ) publiée par O.Z. Dalgaard en 1957 [6]. Cette observation laisse aussi prévoir que la validation d'un gène candidat sera ardue, d'autant plus que les hypothèses sur la nature et la fonction du gène manquent de tout fondement. Reeders et al. ont déjà isolé 5 clones d'ADNc, dont l'étude est en cours, correspondant à des gènes situés dans cette région*.

Une carte génétique précise de cette même région établie par le groupe de M. Breuning à Leiden, Hollande,

\footnotetext{
- Lors d'une ráunion à Leiden (Hollande), début octobre 1990, S.T. Reeders a annoncé que le nombre d'ADNc clonés par son groupe s'élevait à 12 . En revanche, la liaison rapportée entre PKD 2 et un marqueur du chr 2 n'a pas été retrouvée par doux autres groupes (résultats non publiés).

$\mathrm{m} / \mathrm{s} n^{\circ} 9$, vol. 6 , novembre 90
}

avec 16 sondes [7] a montré que celle-ci est aussi caractérisée par une inversion du rapport des fréquences de recombinaison homme/femme par rapport à ce qui est observé sur la majeure partie du génome. En outre, les résultats de quatre groupes italiens portant sur 55 familles semblent montrer un déséquilibre de liaison avec deux sondes distales proches de PKD1, pGGG1 et CMM65. Classiquement, ce type de résultat indique, d'une part, que les marqueurs sont effectivement proches du locus morbide et, d'autre part, qu'une mutation ancestrale relativement récente est à l'origine de la majorité des cas. Un groupe danois dirigé par Søren Nørby, à Copenhague, a établi un premier linkage entre le locus PKD2 et un marqueur génétique hyperpolymorphe du bras court du chromosome 2 , avec un lod-score $\mathrm{Z}=2,12$ pour $\theta=0,108$. Cela signifie que la probabilité de l'existence de cette liaison génétique est 132 fois supérieure à la probabilité de sa non-existence, à une distance de $10,8 \%$ de recombinaison. Ce résultat doit évidemment être vérifié sur la dizaine de familles de type PKD2 connues à ce jour*.

L. Guay-Woodford et al. ont montré, dans un très élégant travail, que le gène responsable de la mutation $c p k$ de la souris, qui constitue un modèle semblable à la polykystose récessive humaine (ARPKD) est situé sur le chromosome 12 murin, synténique de la région $2 \mathrm{p} 23-2 \mathrm{p} 25$ humaine. S'il s'avérait que PKD2 et ARPKD étaient effectivement situés sur le même chromosome (chr 2), cela ouvrirait le champ à de très nombreuses hypothèses qu'il faudra évidemment tester une fois ces linkages confirmés.

Cette accumulation de résultats montre que, après avoir été un peu le parent pauvre de la recherche en génétique humaine, le domaine des néphropathies héréditaires de l'adulte et de l'enfant (voir aussi la découverte très récente $d u$ gène $d u$ syndrome d'Alport lié au chromo- some $\mathrm{X}, \mathrm{m} / \mathrm{s}, n^{\circ} 7$, vol. 6, p. 710) est devenu un axe de recherche majeur. Il faut cependant se garder de tout optimisme excessif, comme le montre la longue quête, encore infructueuse à ce jour, du gène de la chorée de Huntington

\section{Lucien Bachner}

ICGM, Inserm U. 129, laboratoire de biochimie génétique, hôpital Cochin, 24, rue du Faubourg-Saint-Jacques, 75014 Paris, France.

\section{RÉFÉRENCES}

1. Reeders ST, Breuning MH, Davies KE, et al. A Highly polymorphic DNA marker linked to adult polycystic kidney disease on chromosome 16. Nature $1985 ; 317542-4$.

2. Romeo G, Costa G, Catizone L, et al. A second genetic locus for autosomal dominant polycystic kidney disease. The Lancet 1988 ; ii 8-11.

3. Kimberling WJ, Fain PA, Kenyon JB, Goldgar DBS, Sujansky E, Gabow PA. Linkage heterogeneity of autosomal dominant polycystic kidney disease. $N$ Engl $J$ Med 1988 ; 319 : 913-8.

4. Brissenden JE, Roscoe JM, Silverman M. Linkage heterogeneity in autosomal dominant polycystic kidney disease. $A m ~ J$ Hum Genet $1989 ; 45: 132$.

5. Harris PC, Barton NJ, Higgs DR, Reeders ST, Wilkie OM. A long range restriction map between the $\alpha$-globin complex and a marker closely linked to the polycystic kidney disease 1 (PKD1) locus. Genomics 1990 ; 7 : 195-206.

6. Dalgaard OZ. Bilateral polycystic kidney disease of the kidneys: A follow-up of two hundred eighty four patients and their families. Acta Med Scand 1957; 328 (Suppl) : 3-255.

7. Breuning $M H$, Snijdewint FGM, Brunner $\mathrm{H}$, et al. Map of 16 polymorphic loci on the short arm of chromosome 16 close to the gene involved in polycystic kidney disease (PKD1). J Med Genet 1990 ; 27 : 603-13. 